\title{
An Overview of Potential Targets for Treating Amyotrophic Lateral Sclerosis and Huntington's Disease
}

\author{
Caroline Zocatelli de Paula, Bruno Daniel Correia Gonçalves, and Luciene Bruno Vieira
}

Laboratório de Neurofarmacologia, Departamento de Farmacologia, Instituto de Ciências Biológicas (ICB), Universidade Federal de Minas Gerais, Campus Pampulha, 31270901 Belo Horizonte, MG, Brazil

Correspondence should be addressed to Luciene Bruno Vieira; lubvieira@ufmg.br

Received 5 December 2014; Accepted 8 April 2015

Academic Editor: Eduardo Candelario-Jalil

Copyright (C) 2015 Caroline Zocatelli de Paula et al. This is an open access article distributed under the Creative Commons Attribution License, which permits unrestricted use, distribution, and reproduction in any medium, provided the original work is properly cited.

\begin{abstract}
Neurodegenerative diseases affect millions of people worldwide. Progressive damage or loss of neurons, neurodegeneration, has severe consequences on the mental and physical health of a patient. Despite all efforts by scientific community, there is currently no cure or manner to slow degeneration progression. We review some treatments that attempt to prevent the progress of some of major neurodegenerative diseases: Amyotrophic Lateral Sclerosis and Huntington's disease.
\end{abstract}

\section{Introduction}

Neurodegenerative diseases are characterized by a slow and progressive loss of neurons in different areas of the central nervous system. Motor neurons are impaired in Amyotrophic Lateral Sclerosis (ALS) as well as thorny striatal neurons in Huntington's disease (HD) [1]. ALS symptoms include a progressive muscle phenotype of spasticity, hyperreflexia, fasciculations, atrophy, and paralysis. The disease is usually lethal within 3-5 years after the diagnosis and in most cases, patients die from respiratory failure [1]. The causes of the disease and the mechanisms causing premature death of motor neurons are not clear. In addition, two types of the disorder are recognized, familial ALS (fALS) and the most common sporadic ALS (sALS) [1].

Currently the only available treatment for ALS is Riluzole, approved for use in 1995. However, Riluzole only slows down the progression of the disease and gives the patient an average of 3 months of extended life span [2]. The exact mechanism of action of Riluzole is unknown; several papers have demonstrated its inhibition of sodium, calcium, potassium, and glutamate currents [3]. The coadministration of Riluzole with other potential therapeutic agents is constantly being assessed and is usually with no promising positive results $[4,5]$. Therefore, the safety of the combination of Riluzole with a second study drug is unpredictable.
Huntington's disease (HD) is an inherited neurodegenerative disorder, characterized by progressive loss of neurons in the whole brain, notably in the basal ganglia and cerebral cortex, resulting in worsening chorea, cognitive and psychiatric disturbances [6]. In the United States, HD occurs in about 1 in 10,000 people. Currently about 30,000 people in the US have HD and up to 200,000 are at risk [7]. $\mathrm{HD}$ is caused by expanded polyglutamine cytosine-adenineguanine (CAG) repeat sequence in the autosomal dominant gene that encodes huntingtin (Htt, 4p16.3) [8]. Mutant huntingtin protein accumulates and produces transcriptional dysregulation, proteasomal, autophagical, mitochondrial, and metabolic dysfunctions, oxidative stress, apoptosis, neuroinflammation, and consequent neurodegeneration, especially in the striatum $[8,9]$. Unlike ALS, no major treatment has been developed specifically for Huntington's disease. Some symptomatic treatments are available for some motor or psychiatric features [10].

Since both ALS and HD are neurodegenerative disorders, the neuronal loss is progressive and leads to severe disability and reduced quality of life and life expectancy [11]. Although multiple factors are involved in the pathogenesis of these neurodegenerative diseases, which have heterogeneous etiology and background, the mechanisms underlying neuronal death are similar, enabling development of drugs that target many disorders [12]. Nervous system damage in response to 
an insult may lead to acute or delayed neuronal death, apoptotic cell death, neuronal degeneration, injury and loss, and gliosis [11]. Thus, neuroprotection can be an important therapeutic intervention that prevents the death of vulnerable neurons and also retracts progression of the disease.

The aim of this review was to discuss possibilities of multimodal and neuroprotective therapies for ALS and HD, employing currently available drugs and potential targets that might be exploited in the future.

\section{Amyotrophic Lateral Sclerosis (ALS)}

Possibly because the pathogenesis of ALS remains mostly unknown, development of treatments that are effective across the spectrum of sporadic and familial ALS has not been achieved. Considerable evidence supports the involvement of mitochondrial dysfunction and oxidative stress, autophagy, apoptosis, and protein aggregation in the pathogenesis of ALS [13]. Several exciting potential targets for drug intervention to lessen neurodegeneration in ALS are in development [14-16]. We described some of the promising candidates for treating ALS.

\subsection{Mitochondrial Function and Oxidative Stress Pathways.} The involvement of mitochondria in oxidative damage, calcium buffering, and signaling of apoptotic pathways puts the organelle in perspective for therapeutic approaches. Questions such as whether mitochondrial dysfunctions are a trigger or a consequence in neurodegenerative disease dynamics are still in debate, and increasing interest and discoveries are in course regarding mitochondrial modulators [17]. Studies in ALS patients present strong evidences of mitochondrial dysfunction [18-20]. Moreover, mutant SOD1 mouse model for ALS shows morphological alterations in motor neurons and skeletal muscle tissue before onset of neurodegeneration symptoms, and similar abnormalities are found in sporadic ALS patients [21-23]. Furthermore mitochondrial alterations are not restricted to SOD1 mutants as demonstrated in TDP43 mutant models [24].

MitoQ is a mitochondrial antioxidant that contains the antioxidant Quinone linked to a lipophilic triphenylphosphonium cation. Researchers showed that MitoQ prolonged life span and the pathology of SOD-knockout Drosophila melanogaster, the enzyme involved in ALS [25]. In addition, the compound has been shown to exert neuroprotective effects in SODG93A mice, slowing functional decline, decreasing oxidative damage and disease progression, and increasing survival [26]. Furthermore, other studies showed that MitoQ preincubation prevented the cell death observed in cultures of motor neurons + SOD-mutant astrocytes alone [27].

2.2. Melatonin. Melatonin is a natural hormone produced and secreted by the pineal gland. It is currently used to increase sleep efficiency and improve cardiovascular system and as an antiaging drug $[28,29]$. Recent researches in experimental models showed positive effects of melatonin in major neurodegenerative disorders such as ALS, Parkinson's disease, Alzheimer's disease, and HD [30]. In ALS, the most promising effects of melatonin are those of blocking apoptotic pathways and reducing oxidative damage. The mechanisms of melatonin antiapoptotic effects are not completely clear, although the mitochondria have been identified as its target [31].

Oxidative damage caused by free-radicals is accepted as a common link between neurodegenerative diseases and melatonin acts scavenging these free-radicals showing antioxidant activity [32]. Experiments demonstrated attenuation of superoxide induced cell death and the modulation of glutamate toxicity by melatonin in vitro NSC-34 motor neurons culture [33].

Other recent study showed an inhibitory activity of melatonin over apoptotic caspase 1/cytochrome c/caspase 3 apoptotic pathway, with melatonin preventing cell death. In ALS SODG93A mice, melatonin inhibited motor neuron death in the ventral horn of spinal cord and delayed disease onset and mortality [34]. The hormone also inhibited Rip2/caspase 1 pathway. In addition, the work had novel findings in showing an association between disease progression and loss of melatonin and melatonin $1 \mathrm{~A}$ receptors (MT1) in spinal cord of mice. These latter are interesting since MT1 receptors are linked to other major neurodegenerative diseases such as HD and Alzheimer's disease [35-37].

Melatonin clinical trials were so far aimed mostly at insomnia and sleep disturbances [38]. However more recently reduced oxidative damage was reported in patients treated with enteral melatonin at high-doses [33]. Melatonin showed well-toleration event at high-doses and crosses easily the blood-brain barrier further supporting its therapeutic potential.

2.3. Protein Aggregation, Altered Autophagy. One of the consequences of the genetic mutations involved in neurodegenerative diseases is the appearance of misfolded mutant protein aggregates [39]. These aggregates can be toxic and can affect organelles such as mitochondria by causing membrane disruption. In this way, some studies support that clearance of these aggregates could be a therapeutic intervention. In ALS, a link between the disease and protein aggregation altered autophagy was initially observed on morphological studies of spinal cord tissues of ALS patients and models, showing increased number of autophagosomes [40-42].

In this way, as evidences aroused that impaired autophagy may play a pathogenic role in neurodegeneration, compounds promoting autophagy started to be tested. Progesterone and trehalose, two autophagy stimulators, have showed promising results in the SOD model, delayed disease onset and prolonged survival, all related to an induction of the autophagic flux $[43,44]$.

Rapamycin, an FDA approved drug, that induces autophagy was tested in the SOD1-G93A model with negative results, enhancing the rate of disease progression and motor neuron death $[45,46]$. Thus Rapamycin may be, in part, detrimental in ALS due to its immunosuppressive action [45]. However, there are recent data showing that Rapamycin enhances autophagy in immunodeficient mice avoiding the progression of ALS [47]. These authors used 
a strategy to avoid the immunosuppressive effect of Rapamycin and so observed its action on increasing autophagy. Then in this case Rapamycin slowed down the progression of ALS [47].

In addition to the previously mentioned effects, the hormone melatonin has also demonstrated neuroprotective activity through enhanced autophagy [48, 49]. Melatonin protected against apoptosis via a mitochondrial pathway, reducing caspase 3 activity, cytochrome c release, and increasing LC3-II/LC3-I levels, an autophagy marker, as demonstrated by Chen et al. [48]. The granular corneal dystrophy type 2 hallmark is linked to impaired autophagic degradation of mutant protein deposits [49]. Choi et al. also demonstrated that melatonin activates autophagy via the mammalian target of Rapamycin pathway (mTOR). Melatonin has showed positive effects in autophagosome formation and maturation, and its cotreatment with Rapamycin had additive effects in autophagic clearance of mutant protein aggregates, in comparison to either drug alone [49]. Although Rapamycin effects of suppression of protective immune responses and enhancement of protective autophagy can cancel each other out in general health improvement [45], these findings suggest that more specific targeting of autophagy with drugs that produce less side effects holds potential for halting disease and neurodegeneration. For that matter melatonin treatment can be a more efficient option with fewer side effects. Moreover it could be possible that cotreatment with melatonin and lower dosages of Rapamycin would increase efficacy while maintaining Rapamycin-induced immunosuppression controlled.

\subsection{Targeting the Endocannabinoid System. The endocanna-} binoid system is recognized as playing a major role in modulating various processes in the body. Increasing evidences suggest an antioxidant, anti-inflammatory, neuroprotective, and other activities such as improving appetite, anxiety, and depression related to endocannabinoids and cannabinoid therapies [50]. However, there are still major controversies regarding therapeutical applications of the endocannabinoid system, such as whether therapeutic effects are achieved by direct agonism/antagonism of cannabinoid receptors or by modulating the endocannabinoid system tonus, by reducing degradation of natural cannabinoids such as anandamide $[50,51]$.

In ALS, the antioxidant, anti-inflammatory, and neuroprotective activities of cannabinoids are expected to improve ALS symptoms. First surveys assessed marihuana usage in ALS patients and associated it with improvements of appetite, depression, pain, spasticity, and drooling [52].

In ALS SOD1-G93A mice, scientists assessed the effects of cannabinol, a nonpsychotropic compound of the plant. Positive effects regarding disease progression and delay of approximately 2 weeks of disease onset were observed, providing evidence of cannabinoid treatment for ALS [53]. Further evidence was found, showing that cannabinol, another phytocannabinoid, also exerts positive effects of normalizing mitochondrial dynamics associated with caspase 3, DNM1L, and synaptophysin levels in models [54]. More recently, a combination of phytocannabinoids called Sativex was tested in SOD1-G93A mice with moderately positive results [55]. All of these emerging findings point towards neuroprotective and antiapoptotic activities of the cannabinoids in neurodegenerative processes. Better results are expected by the design of more specific compounds, acting on specific populations of cannabinoid receptors, as suggested by discoveries of specific neuroprotective population of cannabinoid receptors [56].

\section{Huntington's Disease}

A neuropathological hallmark of Huntington's disease is the presence of neuronal nuclear inclusions and cytoplasmic aggregates of misfolded mutant huntingtin protein (mHtt) [9]. The mHtt accumulates and produces transcriptional dysregulation, proteasomal, autophageal, mitochondrial, and metabolic dysfunctions, oxidative stress, apoptosis, neuroinflammation, excitotoxicity, and consequent neurodegeneration $[8,9]$. As HD is a genetic disease, affected patients have abnormal huntingtin from the very first moment of the protein's expression, which suggests that neuronal abnormalities might be present since the start. Targeting these pathways might be a cleaver strategy for treating HD.

3.1. Apoptotic Pathways. Neuronal cell death in $\mathrm{HD}$ is associated with neuronal apoptosis, particularly with the initiation of the intrinsic mitochondrial apoptotic pathway [12]. Markers for apoptotic cell death are activated in striatal neurons from both patients with $\mathrm{HD}$ and animals models [57]. Activation of caspases 3 and 9 and release of cytochrome c from the mitochondria into the cytosol are observed both in the brains of patients and animals with HD [58].

Minocycline is an antibiotic, reported to exert neuroprotective activities through caspase 1 , caspase 3 , inducible form of nitric oxide synthase, and p38 mitogen-activated kinase (MAPK). It has good oral bioavailability, tolerability, and crosses blood-brain barrier easily. In ALS models, minocycline delayed disease onset and extended survival. It also inhibited cytochrome $\mathrm{c}$ release, which was demonstrated both in vivo and in isolated mitochondria [59].

Chen et al. reported that the inhibition of caspases 1 and 3 expression by minocycline delayed mortality in R6/2 mice, a model of HD. In addition, minocycline attenuated dopaminergic cell loss and delayed mortality in MPTPtreated mice. Minocycline was taken to human trials for $\mathrm{HD}$ as a promising therapy but failed. Despite its promisor results, it has encountered limitations for its use due toxicity in pharmacological dosages [59]. Moreover, the currently available caspase inhibitors are toxic in pharmacological doses, precluding their immediate use in human studies [12].

3.2. Oxidative Stress. Oxidative stress is characterized by an imbalance between reactive oxygen species (ROS) and antioxidant systems [60]. Interestingly, Chen et al. described a correlation between lipid peroxidation products in plasma and degree of severity in patients with HD [59]. Klepac et al. also reported an occurrence of oxidative stress in HD [61]. 
Dimethyl fumarate (DMF), an essential member of fumaric acid ester (FAE) family, is the active ingredient of BG-12, which has been offered as an effective oral treatment option for patients with relapsing remitting multiple sclerosis (RRMS) [62]. DMF can activate transcription factor nuclear factor (erythroid-derived 2)-related factor 2 (Nrf2). Nrf2 plays an important role of anti-oxidative pathways for tissue protection [63]. There is compelling evidence of the disruption of the Nrf2 system in HD and the contribution of Nrf2 activation to ameliorating oxidative stress and mitochondrial dysfunction in neuronal tissue damage in HD. Jin and colleagues have shown that mHtt disrupts Nrf2 signaling, which contributes to impaired mitochondrial dynamics and may enhance susceptibility to oxidative stress in STHdh (Q111/Q111) cells, striatal cells expressing mHtt [64]. Moreover, DMF treatment leads to an increase in Nrf2 staining in neuronal subpopulations relevant for motor functions, concomitant with elevated Nrf2 immunoreactivity in $\mathrm{R} 6 / 2$ mice, which mimic many aspects of HD. Additional studies in N171-82Q mice, another transgenic mouse model of $\mathrm{HD}$, showed that 2-cyano-3,12-dioxooleana-1,9-dien-28-oic acidethyl amide (CDDO-EA) and 2-cyano-3,12-dioxooleana-1,9dien-28-oic acid-trifluoroethyl amide (CDDO-TFEA) upregulate Nrf2/ARE induced genes in the brain and peripheral tissues and reduce oxidative stress, improving motor impairment and increasing longevity [65].

3.3. Neuroprotective Cell Signaling Pathways. The signaling pathways involved in HD are not yet clearly elucidated [66]. Thus, it is possible that alterations of receptor-mediated signaling pathways could contribute to protection or exacerbation of cell death cascades in the symptomatic and/or presymptomatic phases of HD [67]. Recently, Doria et al., 2015, described the effect of a positive allosteric modulator (PAM) for metabotropic glutamatergic receptor type 5 (mGluR5), named CDPPB (3-cyano-N-(1,3-diphenyl-1Hpyrazol-5-yl)benzamide) [68]. This drug was capable of delaying some symptoms related to $\operatorname{HD}[68,69]$. Chronic treatment of BACHD mice (model of HD) with CDPPB $1.5 \mathrm{mg} / \mathrm{kg}$ for 18 weeks increased the activation of cell signaling pathways, including increased AKT and ERK1/2 phosphorylation, and augmented the BDNF mRNA expression. CDPPB chronic treatment was also able to prevent the neuronal cell loss that takes place in the striatum of BACHD mice and decrease mutant huntingtin aggregate formation [68]. Moreover, CDPPB chronic treatment was efficient to partially ameliorate motor incoordination and to rescue the memory deficit exhibited by BACHD mice. Importantly, no toxic effects or stereotypical behavior was observed upon CDPPB chronic treatment; however the exact CDPPB mechanism of action and more safety tests need to be performed [68].

3.4. Excitotoxicity. Excitotoxicity is known to be an important piece in the development of HD. Thus, antiglutamatergic agents may, therefore, have beneficial neuroprotective effects [70]. One of these agents is tryptophan metabolite kynurenic acid (KYNA), which is an endogenous NMDA receptor antagonist [70]. Although the neuroprotective KYNA shows unaltered levels in mice models, the significant elevation in the concentrations of neurotoxic kynurenine pathway compounds leads to a shift in the metabolism resulting in relative KYNA deficiency [70]. These findings raise the possibility that increasing KYNA effect would be beneficial from a therapeutic aspect. However, higher doses of KYNA have low solubility and poor penetration to blood-brain barrier [71].

Memantine is a noncompetitive N-methyl-D-aspartate (NMDA) receptor antagonist that stabilizes glutamatergic tone. Memantine can attenuate the excitotoxic mechanism [72]. One interesting study examined Memantine as a treatment for patients suffering from Huntington disease. The administration of $20 \mathrm{mg}$ of Memantine/daily significantly improved motor symptoms and improved chorea but failed to improve patient's cognitive, behavioral, functional, or independence ratings [73].

\section{Conclusion}

Neurodegenerative diseases are multifactorial and despite recent progress, basic needs such as the definition of disease biomarkers and molecular mechanisms of neurodegeneration are still to be addressed. No major treatment has been developed specifically for ALS and HD. Some symptomatic treatments are available for some motor or psychiatric features. In the meantime, the revelation of new mechanisms involved in the disease onset and progression gives opportunity for novel approaches in symptomatic treatment. The development or improvement of disease models is also necessary for better assessment of drugs and pathological mechanisms. Multifunctional and multitarget approaches will probably be needed to restore neuronal health when cell dysfunction is present. We must have in mind that multitargeted and combined therapies may be an option in that regard.

\section{Review Criteria}

Articles were selected based on searches of PubMed using a number of different search terms, such as "Neurodegenerative diseases" "Huntington's disease," "Amyotrophic Lateral Sclerosis," "neuroprotection," "neurodegeneration," "pathogenesis," "mitochondria," "apoptosis," "huntingtin," and "autophagy." Only full-text papers written in English and its references were selected.

\section{Conflict of Interests}

The authors declare that there is no conflict of interests regarding the publication of this paper.

\section{Authors' Contribution}

All authors exchanged ideas for the review. Caroline Zocatelli de Paula reviewed the references and prepared the first draft including the reference list. Bruno Daniel Correia 
Gonçalves and Luciene Bruno Vieira reviewed the first draft and provided comments on the references. Luciene Bruno Vieira prepared the final draft. All authors approved the final paper.

\section{Acknowledgments}

This work was supported by Fundação de Amparo a Pesquisa do Estado de Minas Gerais (FAPEMIG), Conselho Nacional de Desenvolvimento Científico e Tecnológico (CNPq), and Pró-Reitoria de Pesquisa da UFMG (PRPq).

\section{References}

[1] National Institute of Neurological Disorders and Stroke, Amyotrophic Lateral Sclerosis (ALS) Fact Sheet, NINDS, 2013.

[2] G. Bensimon, L. Lacomblez, V. Meininger, and ALS/Riluzole Study Group, "A controlled trial of riluzole in amyotrophic lateral sclerosis," The New England Journal of Medicine, vol. 330, no. 9, pp. 585-591, 1994.

[3] M. C. Bellingham, "A review of the neural mechanisms of action and clinical efficiency of riluzole in treating amyotrophic lateral sclerosis: what have we learned in the last decade?" CNS Neuroscience and Therapeutics, vol. 17, no. 1, pp. 4-31, 2011.

[4] L. Dupuis, R. Dengler, M. T. Heneka et al., "A randomized, double blind, placebo-controlled trial of pioglitazone in combination with riluzole in amyotrophic lateral sclerosis," PLoS ONE, vol. 7, no. 6, Article ID e37885, 2012.

[5] S. P. Aggarwal, L. Zinman, E. Simpson et al., "Safety and efficacy of lithium in combination with riluzole for treatment of amyotrophic lateral sclerosis: a randomised, double-blind, placebo-controlled trial," The Lancet Neurology, vol. 9, no. 5, pp. 481-488, 2010.

[6] A. Kent, "Huntington's disease," Nursing Standard, vol. 21, pp. 45-51, 2004.

[7] T. Pringsheim, K. Wiltshire, L. Day, J. Dykeman, T. Steeves, and N. Jette, "The incidence and prevalence of Huntington's disease: a systematic review and meta-analysis," Movement Disorders, vol. 27, no. 9, pp. 1083-1091, 2012.

[8] C. A. Ross and S. J. Tabrizi, "Huntington's disease: from molecular pathogenesis to clinical treatment," The Lancet Neurology, vol. 10, no. 1, pp. 83-98, 2011.

[9] M. DiFiglia, E. Sapp, K. O. Chase et al., "Aggregation of huntingtin in neuronal intranuclear inclusions and dystrophic neurites in brain," Science, vol. 277, no. 5334, pp. 1990-1993, 1997.

[10] F. O. Walker, "Huntington's disease," The Lancet, vol. 369, no. 9557, pp. 218-228, 2007.

[11] F. J. E. Vajda, "Neuroprotection and neurodegenerative disease," Journal of Clinical Neuroscience, vol. 9, no. 1, pp. 4-8, 2002.

[12] R. Djaldetti, N. Lev, and E. Melamed, "Neuroprotection in progressive brain disorders," Israel Medical Association Journal, vol. 5, no. 8, pp. 576-580, 2003.

[13] P. H. Gordon, "Amyotrophic lateral sclerosis: pathophysiology, diagnosis and management," CNS Drugs, vol. 25, no. 1, pp. 1-15, 2011.

[14] G. Siciliano, C. Carlesi, L. Pasquali et al., "Clinical trials for neuroprotection in ALS," CNS and Neurological DisordersDrug Targets, vol. 9, no. 3, pp. 305-313, 2010.

[15] Y. Zhu, A. Fotinos, L. L. Mao et al., "Neuroprotective agents target molecular mechanisms of disease in ALS," Drug Discovery Today, vol. 20, no. 1, pp. 65-75, 2015.
[16] G. Yacila and Y. Sari, "Potential therapeutic drugs and methods for the treatment of amyotrophic lateral sclerosis," Current Medicinal Chemistry, vol. 21, no. 31, pp. 3583-3593, 2014.

[17] H. Kawamata and G. Manfredi, "Mitochondrial dysfunction and intracellular calcium dysregulation in ALS," Mechanisms of Ageing and Development, vol. 131, no. 7-8, pp. 517-526, 2010.

[18] G. Palomo and G. Manfredi, "Exploring new pathways of neurodegeneration in ALS: the role of mitochondria quality control," Brain Research, vol. 1607, pp. 36-46, 2015.

[19] A. C. Ladd, P. M. Keeney, M. M. Govind, and J. P. Bennett, "Mitochondrial oxidative phosphorylation transcriptome alterations in human amyotrophic lateral sclerosis spinal cord and blood," NeuroMolecular Medicine, vol. 16, no. 4, pp. 714-726, 2014.

[20] W. Tan, P. Pasinelli, and D. Trotti, "Role of mitochondria in mutant SOD1 linked amyotrophic lateral sclerosis," Biochimica et Biophysica Acta-Molecular Basis of Disease, vol. 1842, no. 8, pp. 1295-1301, 2014.

[21] C. M. J. Higgins, C. Jung, and Z. Xu, "ALS-associated mutant SOD1 ${ }^{G 93 A}$ causes mitochondrial vacuolation by expansion of the intermembrane space and by involvement of SOD1 aggregation and peroxisomes," BMC Neuroscience, vol. 4, article 16, 2003.

[22] J. Kong and Z. Xu, "Massive mitochondrial degeneration in motor neurons triggers the onset of amyotrophic lateral sclerosis in mice expressing a mutant SOD1," Journal of Neuroscience, vol. 18, no. 9, pp. 3241-3250, 1998.

[23] S. Sasaki and M. Iwata, "Mitochondrial alterations in the spinal cord of patients with sporadic amyotrophic lateral sclerosis," Journal of Neuropathology and Experimental Neurology, vol. 66, no. 1, pp. 10-16, 2007.

[24] W. Wang, L. Li, W.-L. Lin et al., "The ALS disease-associated mutant TDP-43 impairs mitochondrial dynamics and function in motor neurons," Human Molecular Genetics, vol. 22, no. 23, Article ID ddt319, pp. 4706-4719, 2013.

[25] T. Magwere, M. West, K. Riyahi, M. P. Murphy, R. A. J. Smith, and L. Partridge, "The effects of exogenous antioxidants on lifespan and oxidative stress resistance in Drosophila melanogaster," Mechanisms of Ageing and Development, vol. 127, no. 4, pp. 356370, 2006.

[26] E. Miquel, A. Cassina, L. Martínez-Palma et al., "Neuroprotective effects of the mitochondria-targeted antioxidant MitoQ in a model of inherited amyotrophic lateral sclerosis," Free Radical Biology and Medicine, vol. 70, pp. 204-213, 2014.

[27] P. Cassina, A. Cassina, M. Pehar et al., "Mitochondrial dysfunction in SOD1G93A-bearing astrocytes promotes motor neuron degeneration: prevention by mitochondrial-targeted antioxidants," Journal of Neuroscience, vol. 28, no. 16, pp. 41154122, 2008.

[28] R. B. Costello, C. V. Lentino, C. C. Boyd et al., "The effectiveness of melatonin for promoting healthy sleep: a rapid evidence assessment of the literature," Nutrition Journal, vol. 13, no. 1, article 106, 2014

[29] S. D. Paredes, K. A. Forman, C. García, E. Vara, G. Escames, and J. A. Tresguerres, "Protective actions of melatonin and growth hormone on the aged cardiovascular system," Hormone Molecular Biology and Clinical Investigation, vol. 18, no. 2, pp. 79-88, 2014.

[30] X. Wang, "The antiapoptotic activity of melatonin in neurodegenerative diseases," CNS Neuroscience \& Therapeutics, vol. 15, no. 4, pp. 345-357, 2009. 
[31] J. León, D. Acuña-Castroviejo, G. Escames, D.-X. Tan, and R. J. Reiter, "Melatonin mitigates mitochondrial malfunction," Journal of Pineal Research, vol. 38, no. 1, pp. 1-9, 2005.

[32] R. J. Reiter, D. X. Tan, and A. Galano, "Melatonin, exceeding expectations," Physiology (Bethesda), vol. 29, no. 5, pp. 325-333, 2014.

[33] J. H. Weishaupt, C. Bartels, E. Pölking et al., "Reduced oxidative damage in ALS by high-dose enteral melatonin treatment," Journal of Pineal Research, vol. 41, no. 4, pp. 313-323, 2006.

[34] Y. Zhang, A. Cook, J. Kim et al., "Melatonin inhibits the caspase1/cytochrome c/caspase-3 cell death pathway, inhibits MT1 receptor loss and delays disease progression in a mouse model of amyotrophic lateral sclerosis," Neurobiology of Disease, vol. 55, pp. 26-35, 2013.

[35] M. Sánchez-Hidalgo, J. M. Guerrero Montávez, M. D. P. Carrascosa-Salmoral, M. D. C. Naranjo Gutierrez, P. J. Lardone, and C. A. De La Lastra Romero, "Decreased MT1 and MT2 melatonin receptor expression in extrapineal tissues of the rat during physiological aging," Journal of Pineal Research, vol. 46, no. 1, pp. 29-35, 2009.

[36] E. Savaskan, G. Olivieri, L. Brydon et al., "Cerebrovascular melatonin MT1-receptor alterations in patients with Alzheimer's disease," Neuroscience Letters, vol. 308, no. 1, pp. 912, 2001.

[37] X. Wang, A. Sirianni, Z. Pei et al., "The melatonin MT1 receptor axis modulates mutant Huntingtin-mediated toxicity," Journal of Neuroscience, vol. 31, no. 41, pp. 14496-14507, 2011.

[38] A. Carocci, A. Catalano, and M. S. Sinicropi, "Melatonergic drugs in development," Clinical Pharmacology, vol. 6, pp. 127137, 2014.

[39] F. Madeo, T. Eisenberg, and G. Kroemer, "Autophagy for the avoidance of neurodegeneration," Genes and Development, vol. 23, no. 19, pp. 2253-2259, 2009.

[40] A. Li, X. Zhang, and W. Le, "Altered macroautophagy in the spinal cord of SOD1 mutant mice," Autophagy, vol. 4, no. 3, pp. 290-293, 2008.

[41] C. Hetz, P. Thielen, S. Matus et al., "XBP-1 deficiency in the nervous system protects against amyotrophic lateral sclerosis by increasing autophagy," Genes \& Development, vol. 23, no. 19, pp. 2294-2306, 2009.

[42] N. Morimoto, M. Nagai, Y. Ohta et al., "Increased autophagy in transgenic mice with a G93A mutant SOD1 gene," Brain Research, vol. 1167, no. 1, pp. 112-117, 2007.

[43] K. Castillo, M. Nassif, V. Valenzuela et al., "Trehalose delays the progression of amyotrophic lateral sclerosis by enhancing autophagy in motoneurons," Autophagy, vol. 9, no. 9, pp. 13081320, 2013.

[44] J. Kim, T.-Y. Kim, K.-S. Cho, H. N. Kim, and J.-Y. Koh, "Autophagy activation and neuroprotection by progesterone in the G93A-SOD1 transgenic mouse model of amyotrophic lateral sclerosis," Neurobiology of Disease, vol. 59, pp. 80-85, 2013.

[45] X. Zhang, L. Li, S. Chen et al., "Rapamycin treatment augments motor neuron degeneration in SOD1 G93A mouse model of amyotrophic lateral sclerosis," Autophagy, vol. 7, no. 4, pp. 412425, 2011.

[46] A. Bhattacharya, A. Bokov, F. L. Muller et al., "Dietary restriction but not rapamycin extends disease onset and survival of the H46R/H48Q mouse model of ALS," Neurobiology of Aging, vol. 33, no. 8, pp. 1829-1832, 2012.

[47] K. A. Staats, S. Hernandez, S. Schönefeldt et al., "Rapamycin increases survival in ALS mice lacking mature lymphocytes," Molecular Neurodegeneration, vol. 8, no. 1, article 31, 2013.
[48] J. Chen, L. Wang, C. Wu et al., "Melatonin-enhanced autophagy protects against neural apoptosis via a mitochondrial pathway in early brain injury following a subarachnoid hemorrhage," Journal of Pineal Research, vol. 56, no. 1, pp. 12-19, 2014.

[49] S.-I. Choi, K. S. Kim, J.-Y. Oh, J.-Y. Jin, G.-H. Lee, and E. K. Kim, "Melatonin induces autophagy via an mTOR-dependent pathway and enhances clearance of mutant-TGFBIp," Journal of Pineal Research, vol. 54, no. 4, pp. 361-372, 2013.

[50] P. Pacher, S. Bátkai, and G. Kunos, "The endocannabinoid system as an emerging target of pharmacotherapy," Pharmacological Reviews, vol. 58, no. 3, pp. 389-462, 2006.

[51] L. Velayudhan, E. van Diepen, M. Marudkar et al., "Therapeutic potential of cannabinoids in neurodegenerative disorders: a selective review," Current Pharmaceutical Design, vol. 20, no. 13, pp. 2218-2230, 2014.

[52] D. Amtmann, P. Weydt, K. L. Johnson, M. P. Jensen, and G. T. Carter, "Survey of cannabis use in patients with amyotrophic lateral sclerosis," American Journal of Hospice and Palliative Medicine, vol. 21, no. 2, pp. 95-104, 2004.

[53] P. Weydt, S. Hong, A. Witting, T. Möller, N. Stella, and M. Kliot, "Cannabinol delays symptom onset in SOD1 (G93A) transgenic mice without affecting survival," Amyotrophic Lateral Sclerosis and Other Motor Neuron Disorders, vol. 6, no. 3, pp. 182-184, 2005.

[54] V. K. da Silva, B. S. de Freitas, A. da Silva Dornelles et al., "Cannabidiol normalizes caspase 3, synaptophysin, and mitochondrial fission protein DNM1L expression levels in rats with brain iron overload: implications for neuroprotection," Molecular Neurobiology, vol. 49, no. 1, pp. 222-233, 2014.

[55] M. Moreno-Martet, F. Espejo-Porras, J. Fernández-Ruiz, and E. de Lago, " Changes in endocannabinoid receptors and enzymes in the spinal cord of SOD1(G93A) transgenic mice and evaluation of a Sativex-like combination of phytocannabinoids: interest for future therapies in amyotrophic lateral sclerosis," CNS Neuroscience and Therapeutics, vol. 20, no. 9, pp. 809-815, 2014.

[56] A. Chiarlone, L. Bellocchio, C. Blázquez et al., "A restricted population of CB1 cannabinoid receptors with neuroprotective activity," Proceedings of the National Academy of Sciences of the United States of America, vol. 111, no. 22, pp. 8257-8262, 2014.

[57] P. Kumar, H. Kalonia, and A. Kumar, "Huntington's disease: pathogenesis to animal models," Pharmacological Reports, vol. 62, no. 1, pp. 1-14, 2010.

[58] V. O. Ona, M. Li, J. P. G. Vonsattel et al., "Inhibition of caspase1 slows disease progression in a mouse model of Huntington's disease," Nature, vol. 399, no. 6733, pp. 263-267, 1999.

[59] M. Chen, V. O. Ona, M. Li et al., "Minocycline inhibits caspase1 and caspase- 3 expression and delays mortality in a transgenic mouse model of Huntington disease," Nature Medicine, vol. 6, no. 7, pp. 797-801, 2000.

[60] I. Túnez, F. Sánchez-López, E. Agüera, R. Fernández-Bolaños, F. M. Sánchez, and I. Tasset-Cuevas, "Important role of oxidative stress biomarkers in Huntington's disease," Journal of Medicinal Chemistry, vol. 54, no. 15, pp. 5602-5606, 2011.

[61] N. Klepac, M. Relja, R. Klepac, S. Hećimović, T. Babić, and V. Trkulja, "Oxidative stress parameters in plasma of Huntington's disease patients, asymptomatic Huntington's disease gene carriers and healthy subjects: a cross-sectional study," Journal of Neurology, vol. 254, no. 12, pp. 1676-1683, 2007.

[62] M. Hutchinson, R. J. Fox, E. Havrdova et al., "Efficacy and safety of BG-12 (dimethyl fumarate) and other disease-modifying 
therapies for the treatment of relapsing-remitting multiple sclerosis: a systematic review and mixed treatment comparison," Current Medical Research \& Opinion, vol. 30, no. 4, pp. 613-627, 2014.

[63] D.-H. Lee, R. Gold, and R. A. Linker, "Mechanisms of oxidative damage in multiple sclerosis and neurodegenerative diseases: therapeutic modulation via fumaric acid esters," International Journal of Molecular Sciences, vol. 13, no. 9, pp. 11783-11803, 2012.

[64] Y. N. Jin, Y. V. Yu, S. Gundemir et al., "Impaired mitochondrial dynamics and Nrf2 signaling contribute to compromised responses to oxidative stress in striatal cells expressing fulllength mutant huntingtin," PLoS ONE, vol. 8, no. 3, Article ID e57932, 2013.

[65] C. Stack, D. Ho, E. Wille et al., "Triterpenoids CDDO-ethyl amide and CDDO-trifluoroethyl amide improve the behavioral phenotype and brain pathology in a transgenic mouse model of Huntington's disease," Free Radical Biology and Medicine, vol. 49, no. 2, pp. 147-158, 2010.

[66] Y. Sari, "Potential drugs and methods for preventing or delaying the progression of huntington's disease," Recent Patents on CNS Drug Discovery, vol. 6, no. 2, pp. 80-90, 2011.

[67] F. M. Ribeiro, M. Paquet, L. T. Ferreira et al., "Metabotropic glutamate receptor-mediated cell signaling pathways are altered in a mouse model of Huntington's disease," Journal of Neuroscience, vol. 30, no. 1, pp. 316-324, 2010.

[68] J. G. Doria, J. M. de Souza, J. N. Andrade et al., “The mGluR5 positive allosteric modulator, $\mathrm{CDPPB}$, ameliorates pathology and phenotypic signs of a mouse model of Huntington's disease," Neurobiology of Disease, vol. 73, pp. 163-173, 2015.

[69] J. G. Doria, F. R. Silva, J. M. de Souza et al., "Metabotropic glutamate receptor 5 positive allosteric modulators are neuroprotective in a mouse model of Huntington's disease," British Journal of Pharmacology, vol. 169, no. 4, pp. 909-921, 2013.

[70] D. Zádori, G. Nyiri, A. Szonyi et al., "Neuroprotective effects of a novel kynurenic acid analogue in a transgenic mouse model of Huntington's disease," Journal of Neural Transmission, vol. 118, no. 6, pp. 865-875, 2011.

[71] S. Fukui, R. Schwarcz, S. I. Rapoport, Y. Takada, and Q. R. Smith, "Blood-brain barrier transport of kynurenines: implications for brain synthesis and metabolism," Journal of Neurochemistry, vol. 56, no. 6, pp. 2007-2017, 1991.

[72] M. Anitha, M. S. Nandhu, T. R. Anju, P. Jes, and C. S. Paulose, “Targeting glutamate mediated excitotoxicity in huntington's disease: neural progenitors and partial glutamate antagonistmemantine," Medical Hypotheses, vol. 76, no. 1, pp. 138-140, 2011.

[73] W. G. Ondo, N. I. Mejia, and C. B. Hunter, "A pilot study of the clinical efficacy and safety of memantine for Huntington's disease," Parkinsonism and Related Disorders, vol. 13, no. 7, pp. 453-454, 2007. 

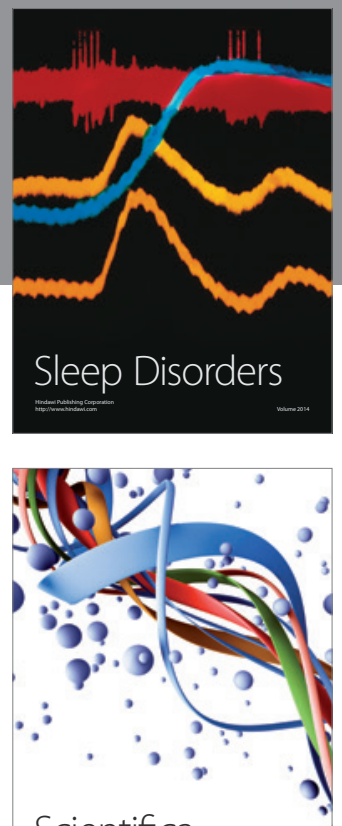

Scientifica
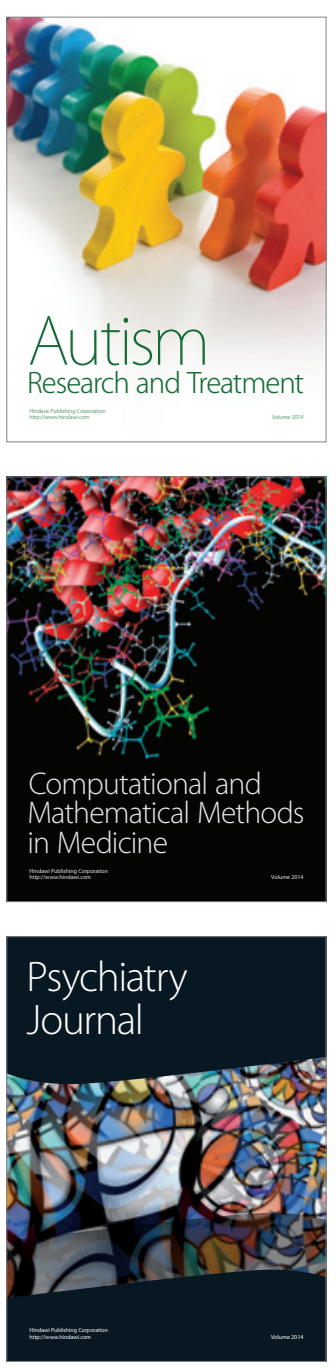
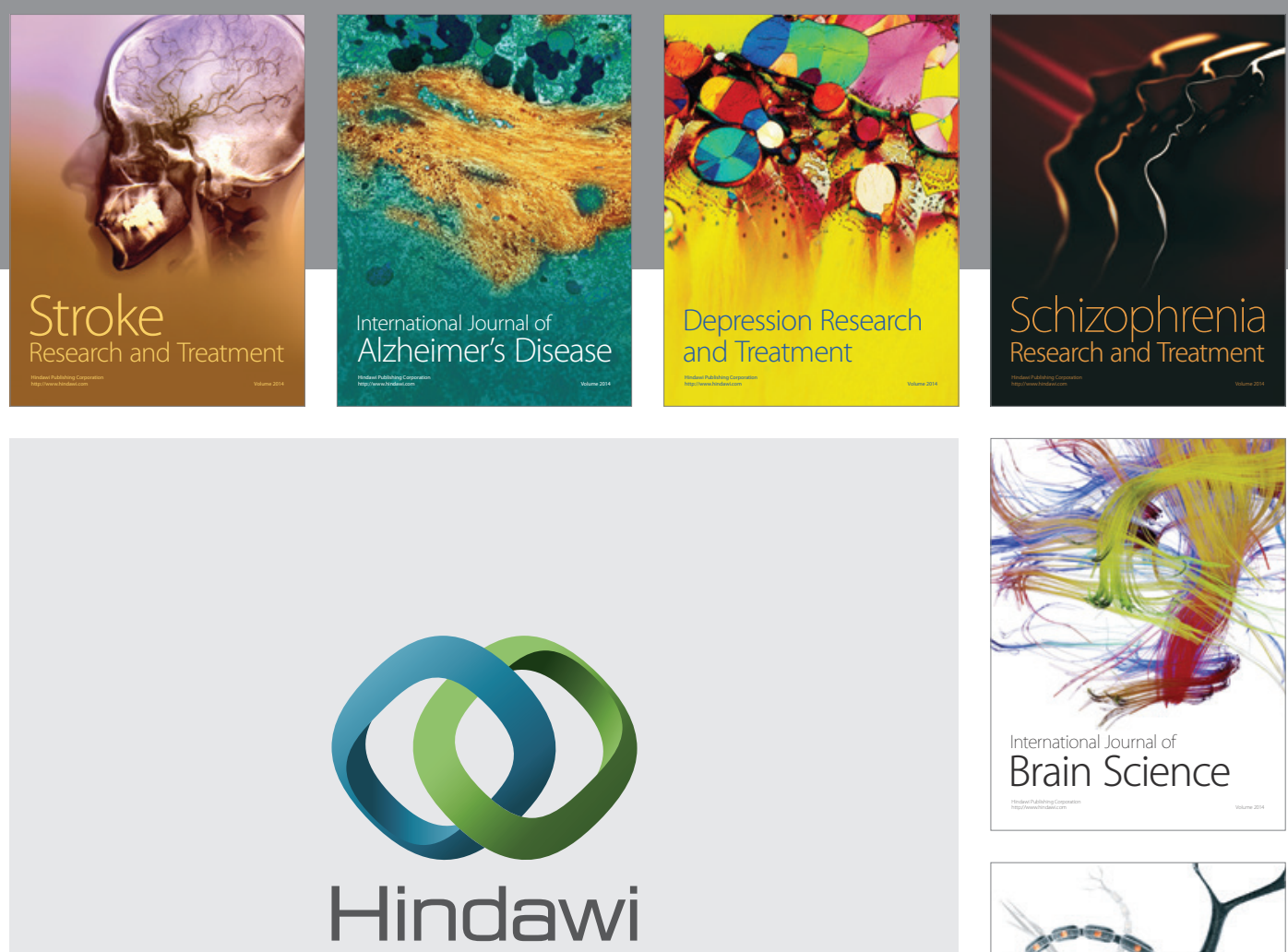

Submit your manuscripts at

http://www.hindawi.com
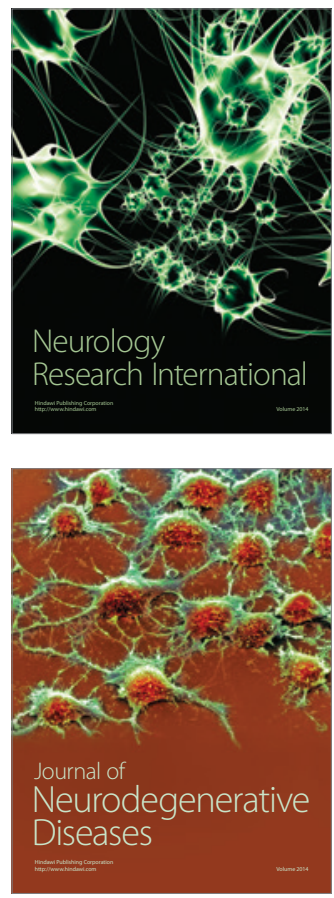

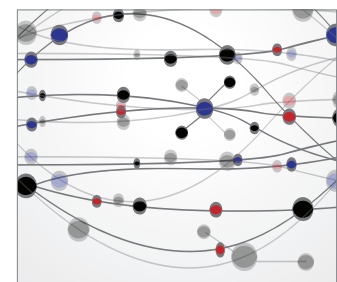

The Scientific World Journal
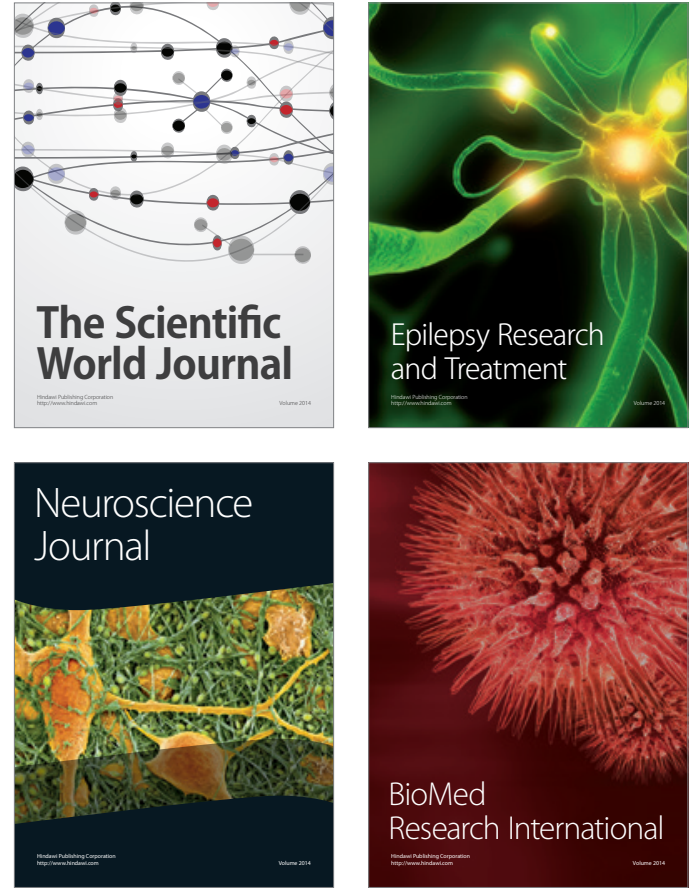

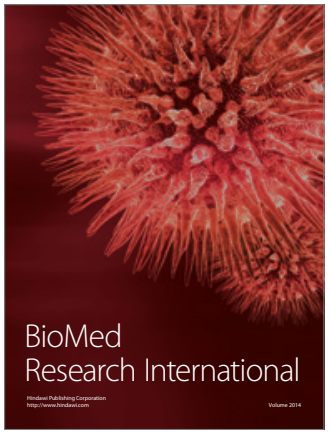

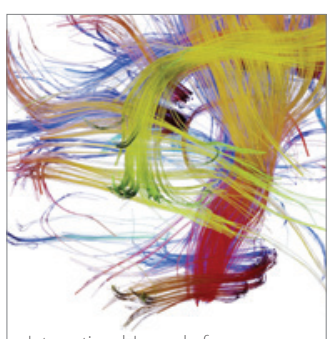

Brain Science

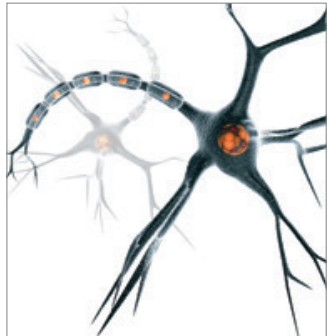

Neural Plasticity
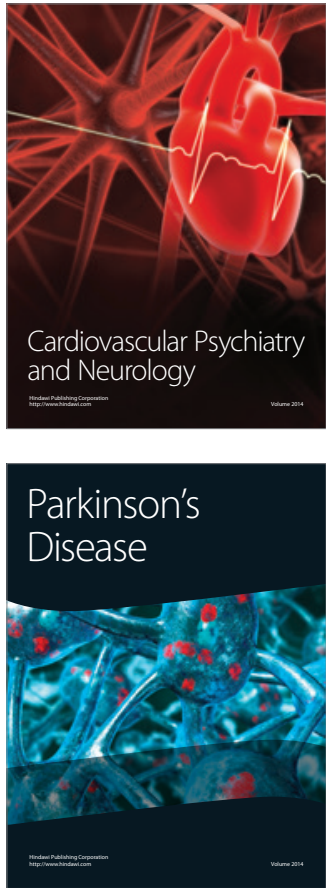